\title{
Aditivos para hormigones, morteros y pastas
}

\author{
DEMETRIO GASPAR TEBAR \\ Dr. en Ciencias Químicas \\ IETCC/CSIC
}

\section{$R E S U M E N$}

En el presente artículo se comenta la importancia que ha adquirido el empleo de los aditivos para hormigones, morteros y pastas, asi como las modificaciones y efectos especificos que producen, lo que ha motivado la necesidad de contar con una normativa española apropiada, cuya realización se encomendó a un Grupo de Trabajo, creado al efecto, de la Comisión Técnica $n{ }^{\circ} 83$ del IRANOR.

Este artículo es el primero de una serie en la que se pretende dar cuenta de los trabajos que sobre normativa de los mencionados aditivos se viene realizando por dicho Grupo de Trabajo.
$S U M M A R Y$

This article comments on the increasing importance of the use of admixtures for concrete, mortars and grouts as well as the specific effects they produce, thus requiring a suitable Spanish Normative, whose performance was taken in charge by a Working Group of the Technical Commission n. ${ }^{\circ} 83$ from the IRANOR, created for this purpose.

This article is first of a serie which intends to give an account of the works, on the Normative of those additives, the Working Group is carrying out.

\section{INTRODUCCION}

El empleo del hormigón en los últimos años, y de un modo especial el incremento que ha experimentado, tanto en la edificación como en las obras civiles, así como:

- los avances de la tecnología en general y la servidumbre que ello impone para el diseño de los correspondientes elementos estructurales,

- el interés de los técnicos de la construcción para mejorar el comportamiento del hormigón en determinados aspectos (trabajabilidad, impermeabilidad, regulacion del tiempo de fraguado, hormigonado en tiempo frí, durabilidad, etc.),

- la aparición y desarrollo de técnicas y procedimientos de puesta en obra para conseguir los objetivos fijados (p.e. hormigón preparado, hormigón proyectado, hormigón bombeado, etc.),

están intimamente ligados al uso de productos adicionados al hormigón en pequeña cantidad; es decir, a los ADITIVOS.

La posibilidad de obtener con los hormigones tradicionales productos capaces de satisfacer las exigencias que, en general, han impuesto los avances tecnológicos, del mismo modo que la de conseguir su estabilidad frente a unas determinadas condiciones ambientes o aumentar el rendimiento en la elaboración del hormigón, es limitada, no permitiendo encontrar, en la mayoría de los casos, una formulación tal que satisfaga las condiciones impuestas para poder fabricar productos más competitivos en los aspectos técnicos y económicos frente a otros materiales. 
Por otra parte, la industria del cemento en los últimos años ha sufrido transformaciones profundas $\mathrm{y}$, fundamentalmente, como consecuencia de la crisis energética ha dado paso a otros cementos fabricados a base de clínker, regulador de fraguado y adiciones (escorias, puzolanas, cenizas volantes, etc.), habiendo conseguido un avance profundo en el conocimiento científico de dichas adiciones y de los cementos que las contienen, asi como en el desarrollo tecnológico de la fabricación y utilización de los mismos.

Del mismo modo, la industria quimica ha experimentado una evolución exponencial en los últimos 25/30 años, de tal modo que se ha conseguido una gama amplia de productos de sintesis y de subproductos que han tenido, también, una influencia decisiva en la industria del hormigón.

Todo ello ha motivado que la industria de aditivos para hormigones, morteros y pastas haya realizado trabajos de investigación y de aplicación que han conducido a la obtención de productos especiales, bien simples o bien fabricados a base de mezclas de distintas materias primas con el fin de tener aditivos polifuncionales, de tal modo que en un período corto de tiempo se ha pasado del empirismo inicial a una industria moderna.

Los aditivos han perdido, en gran parte, su carácter de productos misteriosos con los que se consiguen transformaciones espectaculares; han pasado a ser un componente necesario, en muchos casos, en la fabricación del hormigón, habiendo contribuido eficazmente al desarrollo de la industria del hormigón. Así, hoy día es posible, gracias al uso de estos productos, obtener hormigones con resistencias a compresión elevadas (del orden de $1.000 \mathrm{kp} / \mathrm{cm}^{2}$ ), preparar pastas de cemento muy fluidas (con una relación agua/cemento de $\sim 0,25$ ), poder amasar y transportar el hormigón a distancia regulando el tiempo de aplicación, reducir el tiempo de curado de elementos prefabricados de hormigón, etcétera.

La industria del hormigón en España, así como la de sus componentes y de un modo especial la del cemento, se han desarrollado exponencialmente en los últimos años, tanto como consecuencia de los avances tecnológicos de la industria en general, como de las necesidades derivadas de la crisis energética, de la tecnificación del hormigón y de la mecanización de su fabricación y puesta en obra (centrales de hormigonado, industria de prefabricados, hormigonado a distancia, fabricación de hormigón con bajas relaciones agua/cemento, hormigonado en medios potencialmente agresivos, etc.), lo que ha motivado un aumento considerable del consumo de aditivos, asi como una evolución muy favorable en el nivel de conocimientos en los fabricantes y en los consumidores, que han contribuido al desarrollo de nuevos productos, a la vez que a demandar calidades garantizadas y adecuadas para cada caso concreto.

\section{GENERALIDADES}

Los aditivos para hormigones, morteros y pastas son productos orgánicos y/o inorgánicos que empleados correctamente, y añadidos en pequeña cantidad al hormigón, pueden modificar alguna de sus propiedades en sentido faborable, pero se ha de tener en cuenta que pueden producir otras modificaciones no deseables que deben ser conocidas, fundamentalmente, por los usuarios de estos productos.

Estas modificaciones, favorables o no, pueden ser sensibles a variaciones minimas en la dosificación de dichos productos, en la que, como es lógico, influyen las características de los materiales utilizados en la fabricación del hormigón, teniendo en cuenta que, dadas las características de la industria del hormigón, se producen variaciones inevitables dentro de unos límites determinados.

Así, por ejemplo, la eficacia de ciertos reductores de agua depende del contenido de $\mathrm{C}_{3} \mathrm{~A}$ del cemento; dichos reductores son muy eficaces en cementos de bajo contenido en $\mathrm{C}_{3} \mathrm{~A}$ y apenas si actúan con cementos de elevado contenido (figura 1); esta eficacia también se puede producir en la duración del tiempo de fraguado (figura 2). 
Los aditivos, utilizados correctamente, permiten:

a) Modificar o mejorar:

- la reología del hormigón (mortero o pasta) fresco; es decir, beneficiar la trabajabilidad, disminuir la segregación, etc. (figura 3 ),

- el fraguado y el endurecimiento del cemento (figura 4),

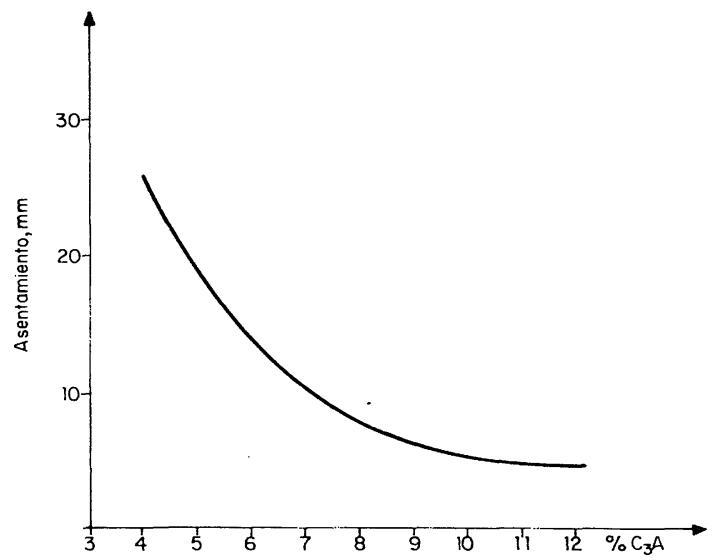

Fig. 1.-Adición del 2 por mil de lignosulfonato de calcio. Trabajabilidad (morteros). Influencia del contenido de $\mathrm{C}_{3} \mathrm{~A}$ del cemento.

(Massazza, F. e Testolin, M.; il cemento, 2, 111, 1980).

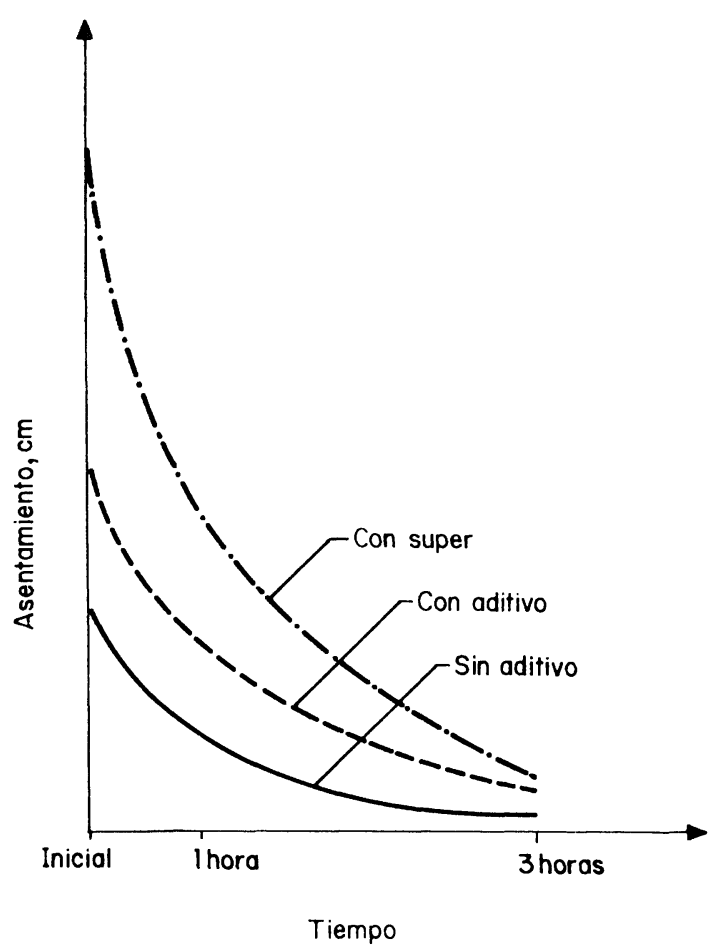

Fig. 3.-Trabajabilidad del hormigon: Influencia del tipo de aditivo.

(Guide to chemical admixtures for concrete. Concrete Society. Technical Report n. 18. London, 1980).
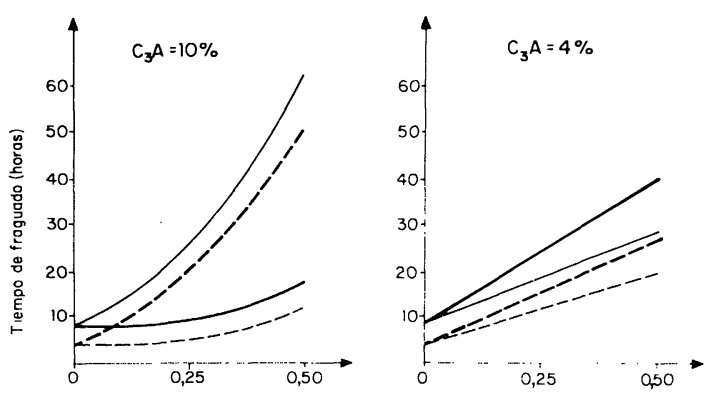

$\%$ de lignosulfonato de calcro, con relación al peso de cemento Fraguado:

$$
\text { ニニニニニ Principio }
$$$$
\overline{\overline{\text { Final }}}
$$$$
\text { Principio del amasado }
$$

- - A los 4 minutos del comienzo del amasodo

Fig. 2.-Adición de lignosulfonato de calcio. Tiempo de fraguado (pasta de cemento). Influencia del contenido de $\mathrm{C}_{3} \mathrm{~A}$ del cemento y del modo de adición del aditivo.

(Venuat, M.: Adjuvants et traitements de mortiers et bétons. Paris, 1971; pág. 159).

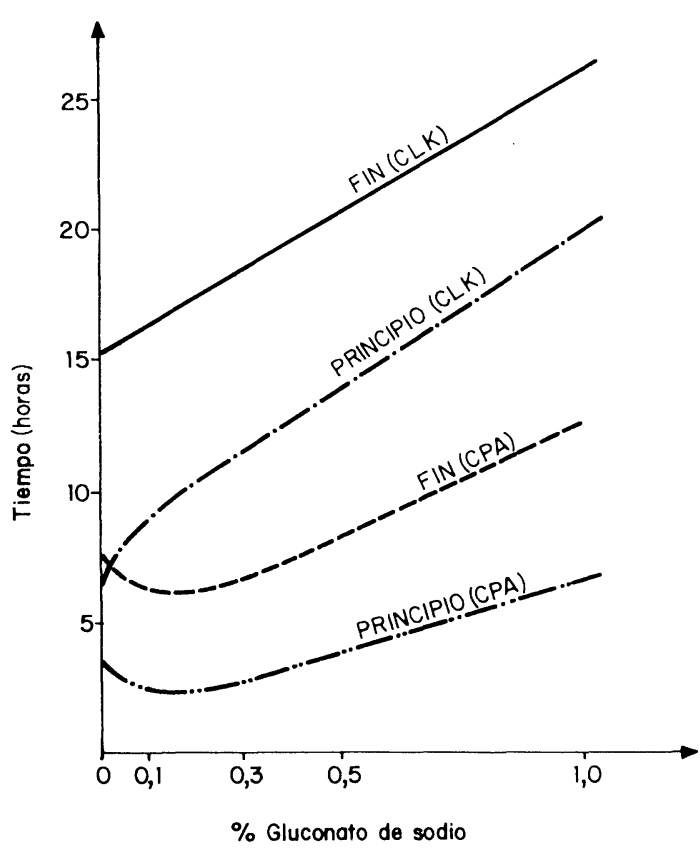

Fig. 4.-Tiempo de fraguado (morteros): Influencia de la cantidad de aditivo.

Morteros (1:3); $\mathrm{a} / \mathrm{c}=0.50 ;$ temperatúra $=20^{\circ} \mathrm{C}$.

Cementos: CLK y CPA.

(Venuat, M.: Adjuvants et traitements de mortiers et bétons. Paris, 1971; pág. 155). 
- las resistencias mecánicas, a ciertas edades, de los hormigones, morteros o pastas (figuras 5 y 6 ),

- las resistencias a acciones físicas, a las acciones mecánicas y a las acciones quimicas (figura 7),

- el contenido de aire, o de otros gases, del hormigón (figura 8).

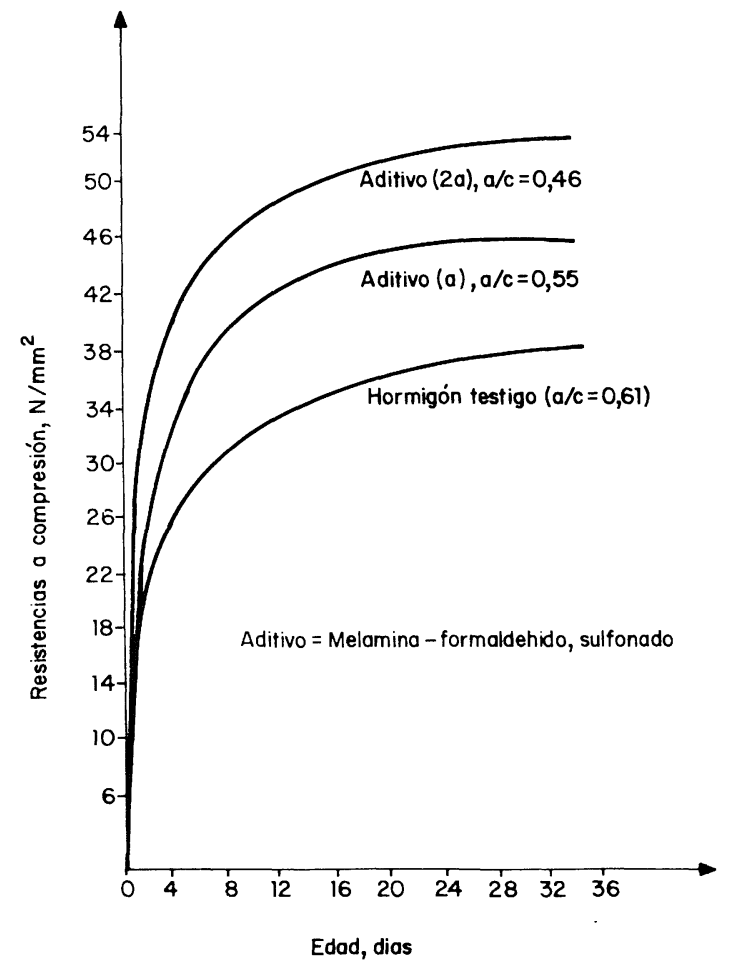

Fig. 5.-Hormigón: Evolución de las resistencias mecánicas, a compresión, en función del tiempo. Influencia de la cantidad de aditivo.

(Massazza, F. e Testolin, M.: Il cemento, 2, 129, 1980).

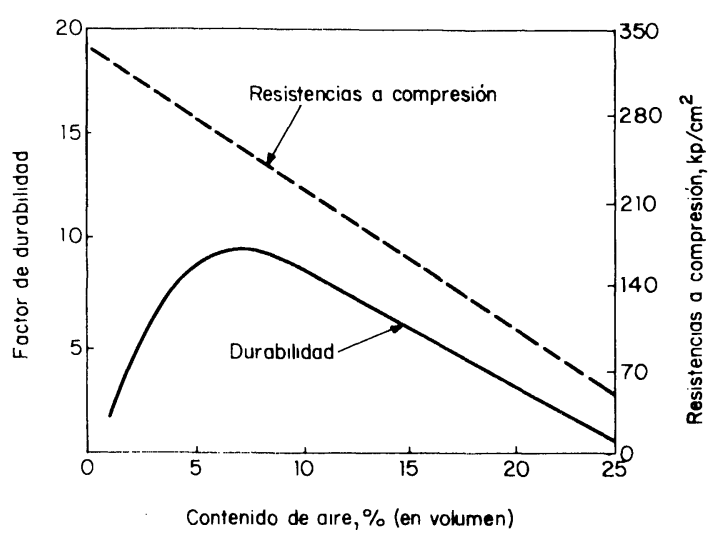

Fig. 7-Hormigón: Durabilidad. Influencia del contenido de aire.

(Mindess, S. and Young, J. F.: Concrete. London, 1981; pág. 179).

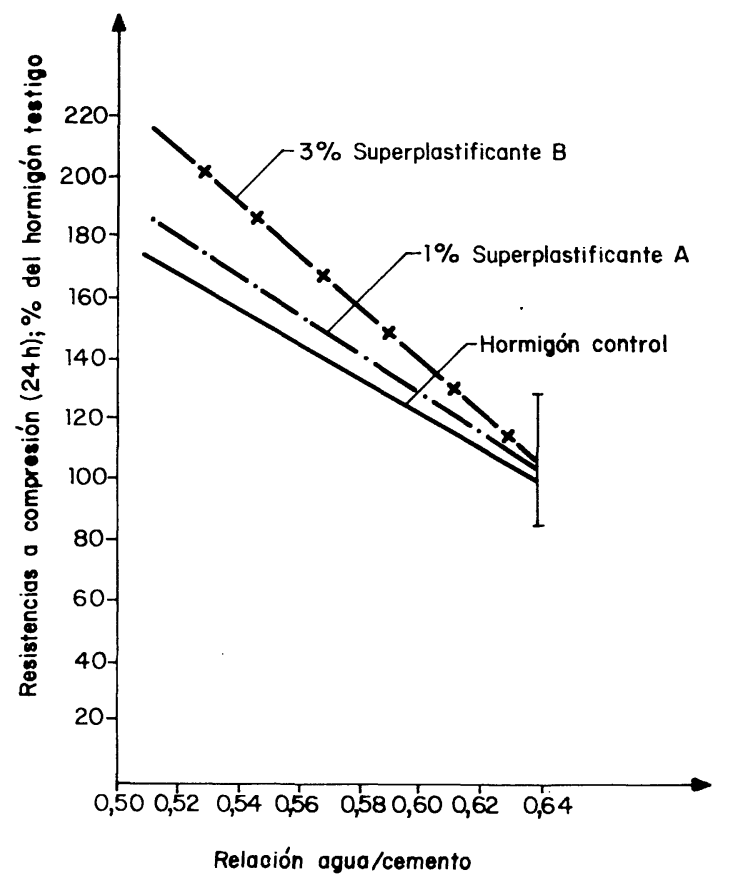

Fig. 6.-Hormigón: Resistencias mecánicas, a compresión. Influencia del tipo de aditivo y de la relación a/c. Hormigón testigo: a/c $=0,64 ; \operatorname{Rc}(24 \mathrm{~h})=8,3 \mathrm{~N} / \mathrm{mm}^{2}=$ $=100 \%$.

(Roberts, M. H. and Adderson, B. W.: Magazine of Concrete Research, 123, 94, 1983).

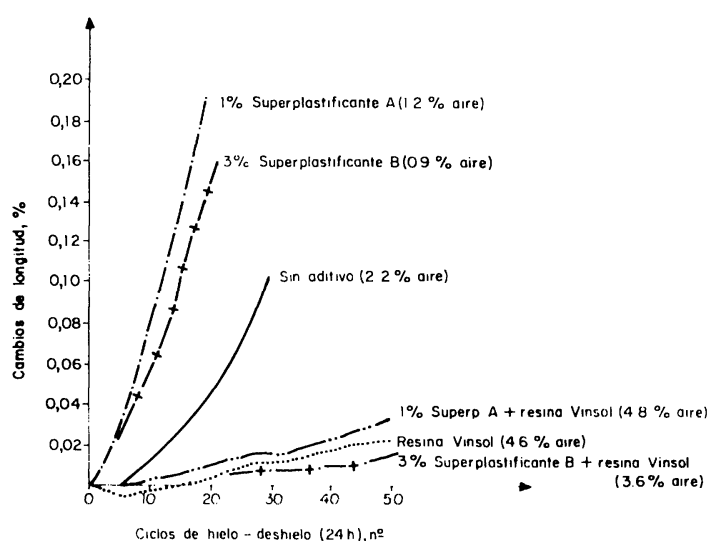

Fig. 8-Hormigón: Resistencia a los ciclos de hielo-deshielo. Influencia del tipo de adtivo y cantidad.

(Roberts, M. H. and Adderson, B. W.: Magazine of Concrete Research, 123, 96, 1983). 
b) Tener una regularidad en la fabricación del hormigón, mortero o pasta y, sobre todo, en su calidad.

c) Ampliar el campo de aplicación del hormigón.

d) Poder disminuir el costo del hormigón (considerando el conjunto de operaciones) por aumentar el rendimiento, por facilitar la puesta en obra, por permitir desencofrar en periodos menores de tiempo, etcétera.

Los aditivos no permiten disminuir, sólo en casos excepcionales, la dosificación de cemento. Así, por ejemplo, no hay que asimilar a los aditivos reductores de agua como reductores de cemento, ya que si bien es cierto que se pueden mantener las resistencias mecánicas cuando las cantidades de cemento experimentan la misma disminución que las del agua -conservando, por consiguiente, constante el valor de la relación agua/cemento-, se pueden modificar otras características del hormigón (mortero o pasta) que pueden ser perjudiciales; p.e., aumentar la permeabilidad, disminuir la capacidad de protección de las armaduras, debilitar la resistencia a los agentes químicos y físicos, etc.

En definitiva, los aditivos no transforman un hormigón malo o defectuoso en otro bueno; modifican, al menos, algunas de las propiedades de los hormigones "buenos" en el sentido positivo deseado.

El empleo de un aditivo no debe alterar en sentido negativo las caracteristicas mecánicas, fisicas o quimicas de los hormigones, morteros y pastas, aun cuando se puede producir una disminución de ellas en determinados casos, en los cuales se deben especificar los descensos que pueden generar dichos aditivos en las normas particulares de cada uno.

Asimismo, el empleo de un aditivo no debe alterar las caracteristicas de las armaduras, tanto del hormigón armado como del pretensado.

La utilización de los aditivos supone tener:

a) un conocimiento apropiado, por parte de los técnicos de la construcción, sobre las posibilidades de estos materiales y, de un modo especial, de su empleo,

b) una información, actualizada, correcta; de tal modo que ante un problema determinado se debe saber:

- si se han de utilizar aditivos,

- cuál se debe emplear, en caso positivo, y

- cómo se debe usar.

Los efectos específicos de los aditivos dependen, por regla general, de diversas variables, entre las que cabe destacar las siguientes:

a) Por parte del cemento:

- tipo de cemento,

- caracteristicas estructurales (composición potencial) de la fracción clínker; especialmente, contenido de $\mathrm{C}_{3} \mathrm{~A}$ y de $\mathrm{C}_{3} \mathrm{~S}$,

- contenido de yeso,

- finura,

- cantidad de cemento,

b) Por parte de los áridos:

- tipo, 
- preparación,

- características: diámetro máximo, granulometria y contenido de finos, porosidad, angularidad, etc.

c) Por parte del aditivo:

- tipo,

- cantidad,

d) Otros factores:

- fundamentalmente, aquellos que afectan a la cinética de la hidratación del cemento (por ejemplo, temperatura, condiciones seguidas para realizar la mezcla, etc.).

De aqui la conveniencia de emplear los aditivos sólo después de conocer sus efectos, de un modo especial, con un hormigón, mortero o pasta definidos (testigos) y, a ser posible, realizados en las condiciones de uso establecidas, ya que:

a) el empleo de un aditivo adecuado puede influir en más de una propiedad del hormigón, mortero o pasta, afectando, en determinados casos, a ciertas características importantes de un modo favorable o adverso,

b) la dosis de los aditivos, junto con las condiciones de empleo y con la naturaleza de los cementos usados en la fabricación del hormigón, mortero o pasta influyen en la acción de un determinado aditivo, de tal modo que puede actuar en un sentido (positivo) o en otro (negativo); de aqui que se imponga controlar rigurosamente la dosificación de los aditivos,

c) la distribución del aditivo en la masa del hormigón debe ser homogénea.

Todo ello ha influido en la necesidad de realizar estudios profundos sobre la acción de los aditivos en el hormigón, tanto desde un punto de vista científico como de aplicación. Con relación a este punto cabe decir que existe una bibliografía muy amplia, recogida en numerosas patentes, revistas, proceedings, etc. en donde se da cuenta de los resultados obtenidos.

Dentro de ésta cabe reseñar por su interés la recogida y publicada en el Capitulo 6 "Admixtures" de Cements Research Progress de los años 1974 a 1982 inclusive (Cements Division. Américan Ceramic Society; Columbus, Ohio). Asimismo, a titulo de ejemplo, para señalar la importancia adquirida por los aditivos, cabe reseñar que en los últimos años han tenido lugar diversos Congresos - Symposiums, entre los que cabe destacar por su importancia los siguientes:

- International Symposium on Admixtures for Mortar and Concrete RILEM/ABEM. Bruxelles; august 30 - septembre 1, 1967.

- Fifth International Symposium on the Chemistry of Cement, IV. Tokyo; october 7-11, 1968.

- Symposium on the Science of Admixtures. London; november 6, 1969.

- The VI International Congress on the Chemistry of Cement. Principal Paper. Moscow; september, 1974.

- International Congress on Admixtures. London; april 16-17, 1980.

- 7. ${ }^{\mathrm{c}}$ Congrès International de la Chimie des Ciments. Rapport principaux, sous-thème VI-1 (vol. I). Thème VI (vol. III). Rapports generauxposters-seminaires (vol. IV). París; Juin 29, Juillet 4, 1980. 
En la actualidad, las revistas relacionadas con los materiales de construcción vienen publicando artículos de esta materia, existiendo algunas que, prácticamente, lo hacen en todos los números.

\section{NORMALIZACION DE ADITIVOS. SITUACION EN ESPAÑA}

La normativa Internacional actual, así como los Pliegos - Instrucciones - Códigos, etc. consideran a los aditivos como uno de los componentes del hormigón. Así, por ejemplo, el Draft Proposal CEN/TC $104 \mathrm{~N} 8$ E del 18-03-83, define al hormigón como “... un material formado por la mezcla de cemento, áridos y agua, y producido por el endurecimiento de la pasta de cemento (cemento y agua); además de estos componentes básicos puede contener aditivos y/o adiciones. El tamaño nominal máximo de los áridos usuales se encuentra comprendido entre 8 y $32 \mathrm{~mm}$ o, en casos especiales, por encima de $32 \mathrm{~mm}$. Si el tamaño máximo de las partículas es de $4 \mathrm{~mm}$ o menos, el material resultante se denomina mortero, no hormigón".

En dichas Normas - Pliegos - Prescripciones - Códigos... se especifican las caracteristicas de los componentes básicos del hormigón, es decir, del cemento, de los áridos y del agua; sin embargo, para los aditivos, únicamente, se señala que antes de su empleo deben verificarse sus efectos por medio de los ensayos correspondientes, hechos en condiciones similares a las reales.

Los aditivos se definen como aquellas sustancias o productos que incorporados a los hormigones, morteros y pastas antes o durante el amasado y/o durante un amasado suplementario, en una proporción no superior al $5 \%$ del peso del cemento (salvo en casos especiales), producen la modificación deseada en estado fresco y/o endurecido de alguna de sus características, de sus propiedades habituales o de su comportamiento.

Esta definición, tomada de la norma española UNE 83.200, coincide con la del Draft ISO 7690 "Concrete, mortars and grouts - Definitions and classification of admixtures" (fecha voto final, 11-02-82), definición que también figura en el mencionado Draft Proposal CEN/TC $104 \mathrm{~N} 8 \mathrm{E}$ "Concrete-Performance, Production, Placing and Compliance Criteria" (pr EN 206), del 18-03-83.

Las adiciones se definen, en dicho Draft Proposal CEN/TC $104 \mathrm{~N} 8$ E, como materiales finamente divididos, p.e. cenizas volantes o escorias granuladas de alto horno, que se pueden añadir al hormigón en cantidades limitadas para mejorar ciertas propiedades o conseguir propiedades especiales. A diferencia de los aditivos, dada su mayor cantidad, se tiene en cuenta su volumen en el diseño de la mezcla.

Conscientes los fabricantes y usuarios españoles de aditivos de que la incorporación al hormigón de dichos aditivos sin control, ni estudios apropiados, puede dar lugar a determinados accidentes, fáciles de eliminar siempre que se utilice el aditivo apropiado en las debidas condiciones y teniendo en cuenta que estos productos no son la panacea universal (es decir, que no constituyen una solución para un hormigón mal estudiado y menos aún revalorizar un hormigón de mala calidad), se creó en el seno de la CT-83 "HORMIGON" del IRANOR, presidida por el Dr. Ing. don Rafael M. a Guitart y de Gregorio, un Grupo de Trabajo (GT-2 "Aditivos") al que se le encomendó la misión de preparar la normativa española correspondiente, dado que, prácticamente, no existian normas españolas relacionadas con esta faceta tan importante, ya que. únicamente, se había normalizado en el año 1958 al cloruro de calcio como producto de adición en los hormigones en las normas UNE 41.113 y UNE 7.138, tituladas "Cloruro cálcico, utilizado como producto de adición en los hormigones" y "Análisis granulométrico y quimico del cloruro cálcico, utilizado como producto de adición en los hormigones", respectivamente.

El mencionado Grupo de Trabajo (GT- 2 "Aditivos"), que empezó sus actividades él dia 23 de marzo de 1981, está constituido por diversos vocales que representan a los sectores de fabricantes y consumidores, asi como los de profesionales, Laboratorios de Ensayo y Centros de 
Investigación $(*)$, habiéndose marcado los siguientes objetivos:

A) Recopilar y estudiar la normativa internacional, asi como la bibliografia relacionada con esta materia.

B) Elaborar los documentos de trabajo, base de los proyectos de norma española (PNE), de acuerdo con los siguientes puntos:

- Primero. Proyecto UNE sobre "Definición y clasificación de aditivos".

- Segundo. Proyectos UNE sobre "Toma de muestras" y "Métodos de ensayo de aditivos".

- Tercero. Proyectos UNE sobre "Materiales y Métodos de ensayo de referencia".

- Cuarto. Proyectos UNE sobre "Especificaciones; condiciones de empleo, así como de suministro y almacenamiento de los aditivos".

- Quinto. Proyectos UNE sobre "Métodos de ensayo de hormigones, morteros y pastas con y sin aditivos".

- Sexto. Proyectos UNE sobre "Métodos de ensayo para identificación rápida de los aditivos en obra".

- Séptimo. Proyectos UNE sobre "Métodos de ensayo para determinar cuali y cuantitativamente a los aditivos en el hormigón endurecido".

Realizando, a ser posible, la experimentación apropiada en cada caso en el seno del Grupo de Trabajo, de tal modo que permita avalar a los diversos proyectos de norma antes de someterlos a la fase de encuesta entre los vocales de la CT-83 "HORMIGON" y posterior fase de encuesta pública. De este modo, se dispondrá de la normativa correspondiente para poder asegurar y garantizar la calidad de los productos fabricados y, a su vez, poder promover y desarrollar criterios de calidad a nivel de marcas, certificados de conformidad, etc., que avalen a los diversos aditivos.

La definición y clasificación de los aditivos se consideró objetivo prioritario por la necesidad de establecer conceptos fundamentales comunes, de tal modo que estuviesen conformes con la normativa internacional, contando, además, con la experiencia de los vocales del Grupo de Trabajo.

Con relación a este punto, conviene señalar que la definición que se tenía de aditivos, considerados como productos de adición en los hormigones, es la que figura en el apartado 2 de la norma UNE 41.113, mencionada anteriormente, "Cloruro cálcico, utilizado como producto de adición en los hormigones", que dice: "De un modo general, se define como productos de adición del hormigón aquellas sustancias distintas del cemento, agua y áridos, que con fines especificos, se incorporan al hormigón durante el proceso de amasado"; definición que, actualmente, no tiene objeto.

(*) En la actualidad forman parte de este Grupo de Trabajo (CT-83/GT-2):

Demetrio Gaspar Tébar; IETcc (Presidente). Gabriel Fernández Fernández; Asland, S. A. (Vicepresidente). José R. Dominguez Bidagor: Halesa (Secretario). Manuel Alvarez Losada; Cons. Sup. Aparejadores. Carlos Chacón Barrilero; ANI. Pedro Ferré Franquet; Sinor-Kao, S. A. Carlos Gajas Fuertes; Texsa. José M. ${ }^{a}$ Garcia San Martin; Seopan. Antonio Garrido Hernández; Laboratorio de Ensayos, Colegio Oficial de Aparejadores y Arquitectos Técnicos de Murcia. Gerardo de Lucas Pérez; Grace, S. A. Gloria Martin Vallejo; INCE. M. ${ }^{\text {a }}$ José Monsalve de la Asunción; Laboratorio d'assaigs Coll. d'Aparelladores i Arq. Técnics de Tarragona. Javier de Nicolás Caparrós; ANL. Gustavo Pezzi Peñalver; Halesa. Rafael Sassot Iribarren; ANI. Gabriel Sémelas Ledesma; Trat. Técnicos del Hormigón, S. A. y Jesús Temiño Fernández; Celite, S. A. 
La finalidad de las normas relacionadas con los "Métodos de ensayo de los aditivos" es para poder identificar desde un punto de vista cualitativo a dichos aditivos y poder determinar cuantitativamente al componente principal de los mismos (compuesto o grupo funcional activo), además de aquellos compuestos o variables que se especifiquen en las normas individuales correspondientes o que se pidan por cualquier circunstancia. En definitiva, se trata de tener métodos de ensayo apropiados para poder identificar a los aditivos con una precisión suficiente, asi como poder disponer de una herramienta imprescindible que permita mantener una calidad en la fabricación y suministro del aditivo, por una parte, y en los productos elaborados (hormigones, morteros y pastas), por otra.

De este modo se consideró que se debían preparar los métodos de ensayo para determinar en los aditivos las siguientes características:

a) de tipo químico: residuo seco a $105 \pm 3^{\circ} \mathrm{C}$, de los aditivos líquidos; pérdida de masa a $105 \pm 3^{\circ} \mathrm{C}$, de los aditivos sólidos; pérdida por calcinación a $1.050 \pm 25^{\circ} \mathrm{C}$; contenido de agua no combinada; residuo insoluble en agua destilada; residuo insoluble en agua saturada de cal; contenido de halogenuros; contenido de reductores; contenido de sulfatos; contenido de nitritos; contenido de nitratos y contenido de fosfatos.

b) de tipo fisicoquímico: peso especifico de los aditivos líquidos; densidad aparente de los aditivos sólidos; pH; punto de congelación de los aditivos líquidos; superficie específica de los aditivos sólidos; estabilidad de espuma; tensión superficial.

c) de identificación: espectro infrarrojo; estudio por rayos $\mathrm{X}$, otros ensayos (a estudiar en su día).

La preparación de los proyectos de normas sobre "Métodos de ensayo con pastas" se pensaron con objeto de poder proporcionar a los usuarios de aditivos una información veraz y para que, a su vez, permitan controlar la idoneidad y eficacia de un aditivo dado, de tal modo que para un cemento con unas características determinadas se pueda conocer la influencia de distintas proporciones de dicho aditivo en pastas, que también se preparen sin aditivo, sobre: la cantidad de agua necesaria para obtener la pasta normal; el tiempo de fraguado; la estabilidad de volumen; el calor de hidratación; la estabilidad de color, etc., para pastas que tengan la misma y distinta consistencia que la del cemento sin aditivo, objeto de estudio.

Por otra parte, estos métodos de ensayo son necesarios para poder conocer el comportamiento de los diversos aditivos con los cementos testigo que se señalen en su momento.

Del mismo modo que en el caso de los proyectos UNE sobre los "Métodos de ensayo con pastas", los proyectos de normas sobre "Métodos de ensayo con morteros y hormigones" son necesarios para poder proporcionar una información veraz a los usuarios de aditivos y para poder controlar su eficacia. Para la elaboración de estos métodos de ensayo se tendrán en cuenta los referentes a morteros y hormigones sin aditivos, ya que en gran parte están normalizados.

Los métodos de ensayo cuya preparación se ha previsto realizar, son los siguientes:

a) en morteros y hormigones frescos, con y sin aditivos: determinación de la plasticidad (consistencia) de los morteros y hormigones (con igual contenido de agua); determinación del contenido de agua; determinación del tiempo de fraguado; determinación del contenido de aire; determinación de la retracción, a distintas edades; determinación de la exudación en morteros y hormigones preparados para todas las determinaciones, excepto para la primera, con la misma plasticidad.

b) en morteros y hormigones endurecidos preparados con y sin aditivos, con la misma plasticidad: determinación de las resistencias mecánicas a flexión, tracción y/o compresión 
(a distintas edades); determinación de la variación de dimensiones (a distintas edades); determinación de la absorción de agua (a distintas edades); determinación de la permeabilidad bajo presión; determinación de la resistencia a los ciclos de hielo-deshielo; determinación de la resistencia química.

Estos métodos de ensayo con morteros y hormigones son necesarios, como en el caso de las pastas, para conocer el comportamiento de los diversos aditivos con los cementos testigo que se señalen en su momento. Por otra parte, estos métodos de ensayo son necesarios para realizar los estudios correspondientes sobre morteros y hormigones, con y sin aditivos, hechos en condiciones análogas a las de obra.

La finalidad de los proyectos de normas sobre "Especificaciones de los aditivos; condiciones de empleo, así como de suministro y almacenamiento" es definir las características de los distintos aditivos de acuerdo con su función principal normalizada, teniendo en cuenta los diversos parámetros que intervienen en su eficacia y en sus efectos secundarios, así como definir: las condiciones de preparación y modo de introducción del aditivo en la mezcla (hormigón, mortero o pasta); la dosificación recomendada, en función de la naturaleza del cemento y de la cantidad de dicho cemento, que se ha de emplear en la elaboración del hormigón; las prescripciones particulares según la naturaleza de los áridos, presencia de materiales muy finos y cantidad; la incompatibilidad (posible) con ciertos cementos o con otros aditivos; la influencia de las condiciones atmosféricas, transporte del hormigón, puesta en obra, etc.; las condiciones de suministro y almacenamiento del aditivo, es decir, el grado de estabilidad del producto antes de su empleo en la fabricación del hormigón, mortero o pasta; el manejo del producto; precauciones que se deben tomar, en su caso.

Todo ello, avalado por la bibliografía y, sobre todo, por la experiencia que se tiene, tanto por los fabricantes como por los consumidores de aditivos.

Por medio del grupo de los proyectos de normas sobre "Métodos de ensayo para la identificación rápida de los aditivos en obra" se pretende garantizar al usuario que el aditivo suministrado a lo largo del tiempo que dure la obra mantiene las especificaciones fijadas. Para ello, se debe disponer de métodos de ensayo rápidos, que sean fáciles de realizar con una instrumentación mínima; como es, por ejemplo, la determinación de las características organolépticas (color, olor, estado, etc.); $\mathrm{pH}$; peso especifico; densidad; solubilidad; etc.

La realización de estos métodos de ensayo, por sus condiciones y por su finalidad, implican, en principio, grandes dificultades.

Con frecuencia, se presenta la necesidad de tener que determinar la presencia o no de un determinado aditivo en un hormigón endurecido, así como estudiar, en ciertos casos, si además existe una sobredosis accidental (séptimo objetivo). De aquí, la conveniencia de disponer de métodos de ensayo para poder identificar y determinar cuantitativamente a los aditivos en los hormigones endurecidos, contando con las dificultades que ello lleva consigo; unas, inherentes al aditivo por su naturaleza y por las cantidades utilizadas (menores del $1 \%$ de la masa del hormigón) y otras, derivadas de las caracteristicas de los componentes del hormigón y del propio hormigón, así como de su historia.

En definitiva, es un problema que preocupa a los técnicos de la construcción y de los aditivos; problema que se piensa estudiar por parte del Grupo de Trabajo una vez que se hayan alcanzado los minimos admisibles en los objetivos anteriores.

(Continuará) 